\title{
USOS DE LOS ACELERÓMETROS EN FISIOTERAPIA: UNA REVISIÓN DE LA LITERATURA
}

\section{USES OF ACCELEROMETERS IN PHYSIOTHERAPY: A REVIEW OF THE LITERATURE}

\author{
Alfonso Mantilla, $\mathrm{JI}^{1}$ \\ ${ }^{1}$ Alfonso Mantilla, Jl. Fisioterapeuta, Universidad del Rosario, Colombia, \\ josealfonso25@hotmail.com
}

Código UNESCO: 321311 . Fisioterapia

Clasificación Consejo de Europa: 14. Fisioterapia y Rehabilitación

Recibido el 9 de enero de 2017

Aceptado el 28 de mayo de 2017

Correspondencia:

Jose Iván Alfonso Mantilla

josealfonso25@hotmail.com

\section{RESUMEN}

En la actualidad, los dispositivos electrónicos se están convirtiendo en herramientas de innovación tecnológica en ciencias de la salud. Entre los nuevos dispositivos se encuentran los acelerómetros, los cuales son sensores electrónicos encargados de la medición de la aceleración durante el movimiento. En fisioterapia, el uso de la tecnología como medio de evaluación e intervención está tomando rumbos encaminados a desarrollar e implementar protocolos estandarizados y validados para la utilización de estos equipos, por tal motivo el objetivo de este artículo es realizar una revisión sistemática de la literatura con relación al uso de los acelerómetros en rehabilitación.

PALABRAS CLAVE: Acelerometría, actividad física, rehabilitación

\section{ABSTRACT}

At present, electronic devices are becoming tools of technological innovation in health sciences. Among the new devices are accelerometers, which are electronic sensors responsible for measuring acceleration during movement. In physiotherapy, the use of technology as a means of evaluation and intervention is taking steps to develop and implement standardized and validated protocols for the use of this equipment, for this reason the objective of this article is to carry out a systematic review of the literature regarding to the use of accelerometers in rehabilitation.

KEY WORDS: Accelerometry, physical activity, rehabilitation 


\section{INTRODUCCIÓN}

En la última década, con los avances investigativos en ciencias de la salud se han creado sistemas electrónicos, robóticos y virtuales para la evaluación e intervención en pacientes con patologías osteomusculares, cardiovasculares y neuromusculares. Adicionalmente, estos sistemas permiten la identificación y almacenamiento de datos con el fin de brindar al usuario un proceso de rehabilitación sistemático e individualizado donde los datos sean una herramienta de seguimiento de objetivos planteados para el cumplimiento de una meta en cuanto el proceso de rehabilitación(1-5). Estos sistemas utilizan estrategias de aprendizaje motor para estructurar los procesos de intervención (1-5). Los procesos de evaluación e intervención son importantes para el entendimiento del estado de salud de un paciente con una patología específica, con estos procesos se garantiza un proceso de rehabilitación exitoso. De hecho, la utilización de pruebas clínicas tradicionales para la evaluación de pacientes es aun utilizada para garantizar evaluaciones objetivas validas y confiables. Sin embargo, se deben desarrollar sistemas electrónicos, robóticos y virtuales para la evaluación e intervención de deficiencias funcionales en pacientes con diversos tipos de patología(3, 5, 6).

En la actualidad, uno de los sistemas electrónicos más utilizados en rehabilitación son los acelerómetros, estos sistemas son dispositivos con sensores electrónicos los cuales pueden medir componentes estáticos y dinámicos que se relacionan con características externas e internas del movimiento corporal humano como ángulos articulares o torque $(5,7,8)$. El acelerómetro proporciona una señal eléctrica a través de vibración como una variante de medición física(5, 7, 8).Adicionalmente, los acelerómetros representan sistemas de innovación en tecnología en ciencias de la salud debido a su capacidad de trasporte, almacenamiento y análisis de elementos biomecánicos del movimiento corporal humano(8). Se ha reportado el uso de los acelerómetros en procesos de medición de niveles de actividad física, gasto metabólico, análisis de marcha y aplicaciones clínicas en la medición de temblores en pacientes con enfermedad de Parkinson. De hecho, los acelerómetros se están convirtiendo en una herramienta para la identificación de alteraciones del movimiento corporal humano, esta herramienta permitirá dirigir actividades de intervención basadas desde elementos como la prescripción del ejercicio físico, ejercicio terapéutico, balance y propiocepción para el trabajo con pacientes con alteraciones metabólicas, enfermedades osteomusculares y neuromusculares(9-19).

Con la nueva de tecnología, se garantizan procesos de evaluación cuantificables con seguimiento de resultados a largo plazo, el reto de estas nuevas tecnologías es garantizar validez y confiabilidad en la metodología de aplicación para que se conviertan en los nuevos implementos de evaluación del futuro $(5,7,8)$. Por tal motivo, el objetivo de este artículo es realizar una revisión sistemática de la literatura con relación al uso de los acelerómetros en rehabilitación. 


\section{MATERIAL Y METODOS}

Se realizo una revisión sistemática de la literatura. Se determinaron los siguientes criterios de inclusión y exclusión:

Inclusión: Estudios contemplados del año 2000 al 2016, literatura que contemplara los siguientes términos MeSH: Accelerometry, physical activity, rehabilitation

La literatura se aceptaría en idioma español, ingles y portugués

Se estableció como otro criterio de inclusión que la búsqueda de evidencia se basara en: Fuentes primarias: Estudios ECA, Metaanálisis, casos y controles, estudios de cohorte, revisiones sistemáticas, revisiones de literatura. Fuentes secundarias: Monografías, tesis de grado, libros.

Exclusión: Estudios que su año de publicación fuera inferior al año 2000, que no contemplaran los términos $\mathrm{MeSH}$ establecidos, estudios que no estuvieran disponibles o completos

\section{RESULTADOS}

En la figura 1 se muestra el proceso de extracción de la evidencia identificada en las bases de datos y su proceso de selección.

Se pudo identificar literatura que referencia el uso de acelerómetros en áreas del conocimiento tales como actividad física y salud y rehabilitación.

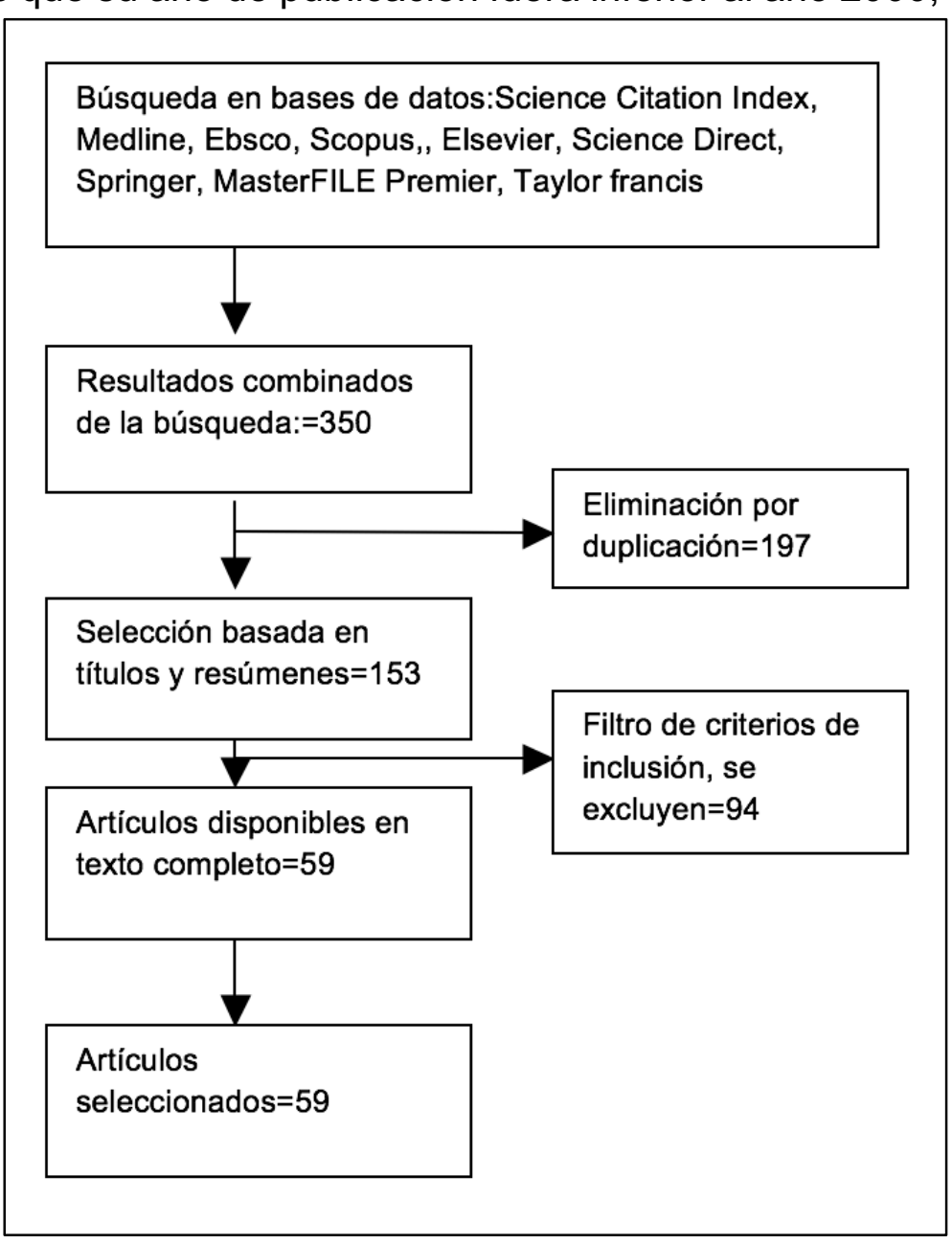

Figura 1. Flujograma de extracción de la evidencia. Fuente de elaboración propia 2016 


\section{Actividad física y salud}

Se pudo identificar la acelerometría como un proceso para la medición del nivel de actividad física en niños y adultos. Adicionalmente, este proceso se realiza colocando los acelerómetros en lugares específicos que midan la aceleración ante la realización de actividad física. De hecho, los procesos de investigación están optando por la utilización de acelerómetros para la medición de niveles de actividad física con el fin de identificar riesgos cardiovasculares y metabólicos para la salud(20-27). Se ha reportado que los niveles de actividad física han disminuido produciendo enfermedades metabólicas como sobrepeso y obesidad en niños y niñas con bajos niveles de actividad física(17, 28, 29). De igual manera, la medición por acelerometría brinda resultados objetivos en relación a los niveles de actividad física, se demuestra la relación del sedentarismo con la sensibilidad de la insulina, esto se traduce en el desarrollo de diabetes tipo II debido a la relación de la resistencia a la insulina y triglicéridos(30, 31). Adicionalmente, el tiempo de sedentarismo se relaciona con biomarcadores de salud cardiometabólico. Por ejemplo, la circunferencia de la cintura, índice de masa corporal, pliegues cutáneos, presión arterial, lipoproteína, triglicéridos, insulina, proteína $C$ reactiva y glicohemoglobina se alteran por niveles de actividad física reducidos(32-36).

\section{Análisis de movimiento corporal humano}

Actualmente, la tecnología se ha convertido en una herramienta fundamental dentro de las áreas de salud. Por ejemplo, el uso de dispositivos para la tele vigilancia en pacientes con alteraciones del movimiento corporal humano, esto con el fin de detectar anormalidades y generar protocolos de intervención y evaluación basados en resultados objetivos dados por este tipo de tecnología(37-41).

El análisis de marcha es reconocido como un examen que detecta alteraciones cinéticas y cinemáticas durante el proceso de locomoción, con la tecnología actual se cuentan con distintos tipos de herramientas para la medición de la misma, entre los cuales se encuentran los acelerómetros, estos pueden ser usados en pacientes con enfermedades osteomusculares, cardiovasculares y neuromusculares. Se puede hacer uso de los acelerómetros para analizar las afecciones espaciotemporales de la marcha en pacientes con osteoartritis de rodilla, enfermedad de Parkinson, adultos mayores. Adicionalmente, con esta medición se pueden detectar asimetrías en cuanto a la velocidad, acho de paso, cadencia y control de tronco que se relacionen con riesgo de caída y alteración del balance en posición estática y dinámica $(5,11,12,42-50)$. De hecho, la evaluación a través de acelerometría puede detectar alteraciones en la marcha como perturbaciones, temblores, pasos cortos en pacientes con enfermedad de Parkinson y ser una evaluación objetiva para dirigir tratamientos enfocados en la rehabilitación de estos factores y tener un seguimiento cuantitativo de los resultados del paciente debido a que los acelerómetros permiten evaluar en planos frontal, sagital, transversal detectando alteraciones antero posteriores y laterales (51-57). 


\section{DISCUSIÓN}

Los acelerómetros son dispositivos tecnológicos que permiten la medición de niveles de actividad física y parámetros temporoespaciales de la marcha, estos dispositivos son de fácil trasporte y permiten el almacenamiento de datos para el desarrollo de investigaciones en el área de actividad física y salud y rehabilitación. Sin embargo, a lo largo de la revisión hecha se pudieron identificar algunos tópicos que a futuro deben ser revisados para las investigaciones que se vayan a realizar con acelerómetros. En primera instancia, deben generarse protocolos de uso de acelerómetros en ámbitos clínicos y de actividad física, segundo se deben realizar más estudios en relación a la validez y la confiabilidad del uso de los acelerómetros en diversos tipo de población, tercero la calibración de los equipos debe ser esencial para garantizar resultados objetivos en las investigaciones que se realicen con estos equipos. Adicionalmente, se deben seguir haciendo estudios con el fin de hacer esta tecnología asequible para investigaciones en actividad física y salud y rehabilitación con el fin de determinar riesgos cardiometabólico para la salud basados en niveles de actividad física y identificar parámetros de normalidad de marcha en pacientes con enfermedades osteomusculares y neuromusculares con el fin de dirigir actividades de intervención objetivas y ajustadas a metas impuestas por estos sistemas de información $(5,8,58,59)$.

\section{LIMITACIONES Y CAMINOS FUTUROS}

En Colombia, el uso de esta tecnología es aun limitada, es por eso que futuras investigaciones deben estar enfocadas en el desarrollo de acelerómetros de bajo costo que permitan a investigadores desarrollar trabajos de investigación enfocados en la prevención de enfermedades metabólicas y promoción de hábitos y estilos de vida saludable, además realizar investigaciones en rehabilitación con el fin de generar parámetros de normalidad de variables cinéticas y cinemáticas en población colombiana.

\section{CONCLUSIONES}

La tecnología está siendo cada día más utilizada en ciencias de la salud, los acelerómetros son una herramienta que está siendo utilizada en estudios sobre la actividad física y salud y en la evaluación del movimiento corporal humano, se deben seguir utilizando estos dispositivos con el fin de estandarizar protocolos de uso y variables de normalidad en distintos tipos de población.

\section{REFERENCIAS BIBLIOGRÁFICAS}

1. Levac DE, Glegg SM, Sveistrup H, Colquhoun H, Miller P, Finestone $H$, et al. Promoting Therapists' Use of Motor Learning Strategies within Virtual Reality-Based Stroke Rehabilitation. PLoS One. 2016;11(12):e0168311.

2. Dockx K, Bekkers EM, Van den Bergh V, Ginis P, Rochester L, Hausdorff JM, et al. Virtual reality for rehabilitation in Parkinson's disease. Cochrane Database Syst Rev. 2016;12:Cd010760. 
3. Maggioni S, Melendez-Calderon A, van Asseldonk E, Klamroth-Marganska V, Lunenburger $\mathrm{L}$, Riener $\mathrm{R}$, et al. Robot-aided assessment of lower extremity functions: a review. J Neuroeng Rehabil. 2016;13(1):72.

4. Miller LE, Zimmermann AK, Herbert WG. Clinical effectiveness and safety of powered exoskeleton-assisted walking in patients with spinal cord injury: systematic review with metaanalysis. Med Devices (Auckl). 2016;9:455-66.

5. Santos-Lozano A, Garatachea N. Tendencias actuales de la acelerometría para la cuantificación de la actividad física. 2012.

6. Peter O, Fazekas G, Zsiga K, Denes Z. Robot-mediated upper limb physiotherapy: review and recommendations for future clinical trials. Int J Rehabil Res. 2011;34(3):196-202.

7. Fernández JM, Acevedo RC, Tabernig CB. Influencia de la fatiga muscular en la señal electromiográfica de músculos estimulados eléctricamente. Revista EIA. 2007(7):111-9.

8. Torres-Luque G, Fernandez IL, Santos-Lozano A, Garatachea N, Carnero EÁ. Actividad física y acelerometría; orientaciones metodológicas, recomendaciones y patrones. Nutrición Hospitalaria. 2014;31(1):115-28.

9. Dong B, Biswas S, Montoye A, Pfeiffer K. Comparing metabolic energy expenditure estimation using wearable multi-sensor network and single accelerometer. Conf Proc IEEE Eng Med Biol Soc. 2013;2013:2866-9.

10. Compagnat M, Daviet JC, Salle JY, Mandigout S, Meurou J, Bordes J. Accelerometers are they accurate for the estimation of total energy expenditure over activities of daily living in stroke survivors? Ann Phys Rehabil Med. 2016;59s:e74.

11. Clermont CA, Barden JM. Accelerometer-based determination of gait variability in older adults with knee osteoarthritis. Gait Posture. 2016;50:126-30.

12. Gonzalez I, Fontecha J, Hervas R, Bravo J. Estimation of Temporal Gait Events from a Single Accelerometer Through the Scale-Space Filtering Idea. J Med Syst. 2016;40(12):251.

13. Ishii M, Mashimo H. Accelerometer based analysis of gait initiation failure in advanced juvenile parkinsonism: a single subject study. J Phys Ther Sci. 2016;28(11):3252-6.

14. Hickey A, Gunn E, Alcock L, Del Din S, Godfrey A, Rochester L, et al. Validity of a wearable accelerometer to quantify gait in spinocerebellar ataxia type 6. Physiol Meas. 2016;37(11):N105-n17.

15. Weiss A, Sharifi S, Plotnik M, van Vugt JP, Giladi N, Hausdorff JM. Toward automated, at-home assessment of mobility among patients with Parkinson disease, using a body-worn accelerometer. Neurorehabil Neural Repair. 2011;25(9):810-8.

16. Machowska-Majchrzak A, Pierzchala K, Pietraszek S. Analysis of selected parameters of tremor recorded by a biaxial accelerometer in patients with parkinsonian tremor, essential tremor and cerebellar tremor. Neurol Neurochir Pol. 2007;41(3):241-50.

17. Loyen A, Clarke-Cornwell AM, Anderssen SA, Hagstromer M, Sardinha LB, Sundquist K, et al. Sedentary Time and Physical Activity Surveillance Through Accelerometer Pooling in Four European Countries. Sports Med. 2016.

18. Matthews CE, Keadle SK, Troiano RP, Kahle L, Koster A, Brychta R, et al. Accelerometermeasured dose-response for physical activity, sedentary time, and mortality in US adults. Am J Clin Nutr. 2016;104(5):1424-32.

19. Froberg A, Larsson C, Berg C, Boldemann C, Raustorp A. Accelerometer-measured physical activity among adolescents in a multicultural area characterized by low socioeconomic status. Int J Adolesc Med Health. 2016.

20. White $\mathrm{T}$, Westgate K, Wareham NJ, Brage S. Estimation of Physical Activity Energy Expenditure during Free-Living from Wrist Accelerometry in UK Adults. PLoS One. 2016;11(12):e0167472.

21. Van Kann DH, de Vries SI, Schipperijn J, de Vries NK, Jansen MW, Kremers SP. Schoolyard Characteristics, Physical Activity, and Sedentary Behavior: Combining GPS and Accelerometry. J Sch Health. 2016;86(12):913-21.

22. Evenson KR, Wen F, Herring AH. Associations of Accelerometry-Assessed and SelfReported Physical Activity and Sedentary Behavior With All-Cause and Cardiovascular Mortality Among US Adults. Am J Epidemiol. 2016;184(9):621-32.

23. Kerr J, Marinac CR, Ellis K, Godbole S, Hipp A, Glanz K, et al. Comparison of Accelerometry Methods for Estimating Physical Activity. Med Sci Sports Exerc. 2016. 
24. Lockwood J, Jeffery A, Schwartz A, Manlhiot C, Schneiderman JE, McCrindle BW, et al. Comparison of a physical activity recall questionnaire with accelerometry in children and adolescents with obesity: a pilot study. Pediatr Obes. 2016.

25. Weaver RG, Crimarco A, Brusseau TA, Webster CA, Burns RD, Hannon JC. Accelerometry-Derived Physical Activity of First Through Third Grade Children During the Segmented School Day. J Sch Health. 2016;86(10):726-33.

26. Gomersall SR, Ng N, Burton NW, Pavey TG, Gilson ND, Brown WJ. Estimating Physical Activity and Sedentary Behavior in a Free-Living Context: A Pragmatic Comparison of ConsumerBased Activity Trackers and ActiGraph Accelerometry. J Med Internet Res. 2016;18(9):e239.

27. Skender S, Ose J, Chang-Claude J, Paskow M, Bruhmann B, Siegel EM, et al. Accelerometry and physical activity questionnaires - a systematic review. BMC Public Health. 2016;16:515.

28. Corder K, Sharp SJ, Atkin AJ, Andersen LB, Cardon G, Page A, et al. Age-related patterns of vigorous-intensity physical activity in youth: The International Children's Accelerometry Database. Prev Med Rep. 2016;4:17-22.

29. van Bakergem M, Sommer EC, Heerman WJ, Hipp JA, Barkin SL. Objective reports versus subjective perceptions of crime and their relationships to accelerometer-measured physical activity in Hispanic caretaker-child dyads. Prev Med. 2016.

30. Brocklebank LA, Falconer CL, Page AS, Perry R, Cooper AR. Accelerometer-measured sedentary time and cardiometabolic biomarkers: A systematic review. Prev Med. 2015;76:92-102.

31. Henson J, Yates T, Biddle SJ, Edwardson CL, Khunti K, Wilmot EG, et al. Associations of objectively measured sedentary behaviour and physical activity with markers of cardiometabolic health. Diabetologia. 2013;56(5):1012-20.

32. Healy GN, Winkler EA, Brakenridge CL, Reeves MM, Eakin EG. Accelerometer-derived sedentary and physical activity time in overweight/obese adults with type 2 diabetes: crosssectional associations with cardiometabolic biomarkers. PLoS One. 2015;10(3):e0119140.

33. Henson J, Yates T, Edwardson CL, Khunti K, Talbot D, Gray LJ, et al. Sedentary time and markers of chronic low-grade inflammation in a high risk population. PLoS One. 2013;8(10):e78350.

34. Adair LS, Gordon-Larsen P, Du SF, Zhang B, Popkin BM. The emergence of cardiometabolic disease risk in Chinese children and adults: consequences of changes in diet, physical activity and obesity. Obes Rev. 2014;15 Suppl 1:49-59.

35. Edwards MK, Loprinzi PD. Associations between accelerometer-assessed sedentary behavior, physical activity and objectively-measured cardiorespiratory fitness with red blood cell distribution width. Int J Cardiol. 2016;221:755-8.

36. Belcher BR, Moser RP, Dodd KW, Atienza AA, Ballard-Barbash R, Berrigan D. SelfReported Versus Accelerometer-Measured Physical Activity and Biomarkers Among NHANES Youth. J Phys Act Health. 2015;12(5):708-16.

37. Giansanti D, Macellari V, Maccioni G. Telemonitoring and telerehabilitation of patients with Parkinson's disease: health technology assessment of a novel wearable step counter. Telemed J E Health. 2008;14(1):76-83.

38. Giansanti D, Maccioni G, Morelli S. An experience of health technology assessment in new models of care for subjects with Parkinson's disease by means of a new wearable device. Telemed J E Health. 2008;14(5):467-72.

39. Shull PB, Jirattigalachote W, Hunt MA, Cutkosky MR, Delp SL. Quantified self and human movement: a review on the clinical impact of wearable sensing and feedback for gait analysis and intervention. Gait Posture. 2014;40(1):11-9.

40. Godinho C, Domingos J, Cunha G, Santos AT, Fernandes RM, Abreu D, et al. A systematic review of the characteristics and validity of monitoring technologies to assess Parkinson's disease. J Neuroeng Rehabil. 2016;13:24.

41. Maccioni G, Macellari V, Giansanti D. Design and construction of step counters for disable people: preliminary experience at the Italian Institute of Health. Conf Proc IEEE Eng Med Biol Soc. 2007;2007:4927-9.

42. Cahill-Rowley K, Rose J. Temporal-spatial reach parameters derived from inertial sensors: Comparison to 3D marker-based motion capture. J Biomech. 2016.

43. Barden JM, Clermont CA, Kobsar D, Beauchet O. Accelerometer-Based Step Regularity Is Lower in Older Adults with Bilateral Knee Osteoarthritis. Front Hum Neurosci. 2016;10:625. 
44. Hsu CY, Tsai YS, Yau CS, Shie HH, Wu CM. Test-Retest Reliability of an Automated Infrared-Assisted Trunk Accelerometer-Based Gait Analysis System. Sensors (Basel). 2016;16(8).

45. Rezvanian S, Lockhart TE. Towards Real-Time Detection of Freezing of Gait Using Wavelet Transform on Wireless Accelerometer Data. Sensors (Basel). 2016;16(4).

46. Matsumoto H, Hagino H, Osaki M, Tanishima S, Tanimura C, Matsuura A, et al. Gait variability analysed using an accelerometer is associated with locomotive syndrome among the general elderly population: The GAINA study. J Orthop Sci. 2016;21(3):354-60.

47. Ahlrichs C, Sama A, Lawo M, Cabestany J, Rodriguez-Martin D, Perez-Lopez C, et al. Detecting freezing of gait with a tri-axial accelerometer in Parkinson's disease patients. Med Biol Eng Comput. 2016;54(1):223-33.

48. Tomite T, Saito H, Aizawa T, Kijima H, Miyakoshi N, Shimada Y. Gait Analysis of Conventional Total Knee Arthroplasty and Bicruciate Stabilized Total Knee Arthroplasty Using a Triaxial Accelerometer. Case Rep Orthop. 2016;2016:6875821.

49. Yungher DA, Morris TR, Dilda V, Shine JM, Naismith SL, Lewis SJ, et al. Temporal Characteristics of High-Frequency Lower-Limb Oscillation during Freezing of Gait in Parkinson's Disease. Parkinsons Dis. 2014;2014:606427.

50. Wallen MB, Dohrn IM, Stahle A, Franzen E, Hagstromer M. Comparison of pedometer and accelerometer derived steps in older individuals with Parkinson's disease or osteoporosis under free-living conditions. J Aging Phys Act. 2014;22(4):550-6.

51. Matsushima A, Yoshida K, Genno H, Murata A, Matsuzawa S, Nakamura K, et al. Clinical assessment of standing and gait in ataxic patients using a triaxial accelerometer. Cerebellum Ataxias. 2015;2:9.

52. Demonceau M, Donneau AF, Croisier JL, Skawiniak E, Boutaayamou M, Maquet D, et al. Contribution of a Trunk Accelerometer System to the Characterization of Gait in Patients With Mild-to-Moderate Parkinson's Disease. IEEE J Biomed Health Inform. 2015;19(6):1803-8.

53. Howell D, Osternig L, Chou LS. Monitoring recovery of gait balance control following concussion using an accelerometer. J Biomech. 2015;48(12):3364-8.

54. Del Din S, Godfrey A, Rochester L. Validation of an accelerometer to quantify a comprehensive battery of gait characteristics in healthy older adults and Parkinson's disease: toward clinical and at home use. IEEE J Biomed Health Inform. 2015.

55. Godfrey A, Del Din S, Barry G, Mathers JC, Rochester L. Instrumenting gait with an accelerometer: a system and algorithm examination. Med Eng Phys. 2015;37(4):400-7.

56. Godfrey A, Morris R, Hickey A, Del Din S. Beyond the front end: Investigating a thigh worn accelerometer device for step count and bout detection in Parkinson's disease. Med Eng Phys. 2016;38(12):1524-9.

57. Zach H, Janssen AM, Snijders AH, Delval A, Ferraye MU, Auff E, et al. Identifying freezing of gait in Parkinson's disease during freezing provoking tasks using waist-mounted accelerometry. Parkinsonism Relat Disord. 2015;21(11):1362-6.

58. Del Din S, Hickey A, Hurwitz N, Mathers JC, Rochester L, Godfrey A. Measuring gait with an accelerometer-based wearable: influence of device location, testing protocol and age. Physiol Meas. 2016;37(10):1785-97.

59. Corcoran MP, Chui KK, White DK, Reid KF, Kirn D, Nelson ME, et al. Accelerometer Assessment of Physical Activity and Its Association with Physical Function in Older Adults Residing at Assisted Care Facilities. J Nutr Health Aging. 2016;20(7):752-8.

Referencias totales citadas: 59

Referencias citadas correspondientes a la Rev Ib CC Act Fis Dep: 1 